\footnotetext{
1.Institución Educativa Juan José Reyes Patria

andrea.rodriguez09@uptc.edu.co

2. Universidad Pedagógica y

Tecnológica de Colombia
}

Como citar: Rodríguez, A. I., \& Sanabria, N. T. (2021). Interacciones Familiares y Desarrollo Emocional de un Niño de Cinco Años. Educación $Y$ Ciencia, (25), e12662. https:// doi.org/10.19053/0120-7105. eyc.2021.25.e12662

\section{Interacciones Familiares y Desarrollo Emocional de un Niño de Cinco Años}

\author{
Andrea Ivett Rodríguez Daza ${ }^{1}$ \\ Nelsy Teresa Sanabria Jiménez ${ }^{2}$
}

\title{
Resumen
}

Este artículo da cuenta de las interacciones familiares y el desarrollo emocional de un niño de cinco años de Gámeza, Boyacá, a partir de la descripción de las interacciones cotidianas, la interpretación de los estilos parentales y la visibilización de factores que inciden en el desarrollo emocional del niño. Se tuvo en cuenta el método cualitativo con enfoque interpretativo desde el estudio de caso. Las interacciones con la familia se guiaron por técnicas e instrumentos como la observación participante y las entrevistas semiestructuradas. Lo anterior, atendiendo a las categorías inteligencia emocional y estilos parentales. Los aportes de investigaciones y estudios referentes a la temática validan la importancia de las relaciones familiares y sus emociones. Asimismo, los resultados de esta investigación mostraron la incidencia de los estilos parentales en el desarrollo emocional de los nińos, la cual se hizo evidente en la relación entre el nińo y la cultura y experiencias cotidianas entre padres e hijos, que son la base para formar la personalidad. Esta, a su vez, se construye en relaciones participativas y democráticas.

Palabras clave: inteligencia emocional, desarrollo emocional, estilos parentales, familia. 


\title{
Family Interactions and Emotional Development of a Five- Year-Old Children
}

\begin{abstract}
This article presents the family interactions and emotional development of a fiveyear-old boy from Gámeza, Boyacá, from the description of daily interactions, the interpretation of parental styles, and the visibility of factors that affect the child's emotional development. The study used the qualitative method with an interpretative approach from the point of view of the case study. The interactions with the family came to techniques and tools such as participant observation and semi-structured interviews. The above, considering the categories emotional intelligence and parenting styles. The contributions of research and studies on the subject validate the importance of family relationships and their emotions. Also, the results showed the incidence of parental styles on the emotional development of children, which became evident in the relationship between the child and the culture and daily experiences between parents and children, which are the basis for a personality formation. The personality is built on participatory and democratic relations.
\end{abstract}

Key words: emotional intelligence, emotional development, parenting styles, family.

\section{Introducción}

En Colombia, la familia "ha sido quizás la institución más afectada y maltratada por numerosos factores internos y externos; estos en su mayoría, fruto de construcciones culturales hegemónicas que, de un modo u otro, han influido en la formación de nuestro sistema social" (Gutiérrez, 2019, p. 132). Al respecto, Cabrera et al., citados por Henao y García (2009) plantean que

La familia, considerada como el contexto básico de desarrollo humano, es quien tiene la acción socializadora del niño o niña en primera instancia; a través del modelamiento y del tipo de comunicación que se establece dentro de ella, busca llevar a cabo el alcance de metas y pautas comportamentales vinculadas por medio de dos acciones: control y afecto. Lo que busca controlar la familia tiene que ver con el nivel de socialización, la adquisición de patrones de responsabilidad, la evitación de riesgos y fracasos, y la generación de niveles de obediencia (p. 787).

El entorno familiar es el principal agente y escenario socializador donde los niños inician su proceso y desarrollo emocional teniendo en cuenta diferentes estilos parentales como el democrático, permisivo, autoritario e indiferente. Estos permiten caracterizar y conocer actitudes con que las familias asumen la relación de sus hijos con el mundo que los rodea (Capano \& Ubach, 2013). A continuación, se hará una breve descripción de los tipos de estilos parentales a partir de una compilación de 
distintos autores realizada por Jorge y Gonzales (2017).

- Estilo Autoritario: Se caracteriza por la poca comunicación y afectividad del adulto quien está centrado en la perfección, la obediencia y la rigidez de las acciones realizadas por los nińos, por ello, dedica la mayor parte del tiempo a controlar y evaluar los comportamientos de estos; está a favor de las conductas que se castigan o se premian según el comportamiento; percibe la crianza de manera unidireccional, limitando la autonomía e independencia de los infantes.

- Estilo Permisivo: La principal característica es el rechazo del control, el poder instaurado en el comportamiento de los nińos y el uso de castigos. El adulto pretende ser un poco más flexible y ofrecer la posibilidad de tranquilidad desde las responsabilidades y cargas específicas, además, proporciona autonomía en la toma de decisiones y acciones que realiza el nińo. En gran medida son padres positivos, tolerantes y aceptan, al tiempo que justifican, los comportamientos de sus hijos.

- Estilo Democrático: Se caracteriza por la buena comunicación y la gran capacidad de brindar afecto. Los adultos reconocen al nińo como un ser consciente y le precisan responsabilidades acordes a su madurez; reconocen la crianza de manera bidireccional teniendo en cuenta la opinión del niño para crear acuerdos y estrategias en su comportamiento; y generan vínculos de confianza y afecto.

- Estilo Negligente: Se caracteriza por padres que presentan niveles muy bajos en afecto, comunicación, control y exigencias de madurez. También se conoce como padres ausentes.

Las interacciones que se dan en la cotidianidad familiar están permeadas frecuentemente por las emociones. Por lo tanto, se revisa el concepto de Inteligencia Emocional [IE] según modelo de Salovey y Mayer, propuesto en 1990, desde distintas propuestas como la recopilación de la Fundación Botín coordinada por FernándezBerrocal et al. (2015), García (2017) y Bisquerra (2010). Este último plantea que la IE es "la habilidad para manejar los sentimientos y emociones, discriminar entre ellos y utilizar estos conocimientos para dirigir los propios pensamientos y acciones" (p. 128). Ahora bien, Salovey y Mayer consideran un modelo de IE a través de cuatro habilidades básicas. Para el desarrollo de este proyecto se retomarán tres habilidades específicas: la percepción y expresión, la comprensión, y la regulación emocional, que se les atribuirá el nombre de subcategorías. Teniendo en cuenta los distintos aportes acerca de la IE, se trabajará a partir de los planteamientos de Fernández y Extremera (2005). A continuación, se realiza una descripción de cada una de las habilidades:

- Percepción Emocional: Esta habilidad permite revisar y percibir de manera concreta los sentimientos propios y de los demás teniendo en cuenta las diferentes expresiones y rasgos externos como los movimientos corporales, las actitudes, las palabras, entre otros, los cuales denotan distintos estados emocionales. Dentro de esta categoría se encuentran algunas subcategorías como la habilidad para 
identificar emociones propias, habilidad para identificar emociones en otras personas, diseños, arte, etc., a través del lenguaje, sonido, entre otros, y la habilidad para expresar sentimientos propios y necesidades asociadas a los mismos.

- Comprensión Emocional: Es la habilidad para conocer y analizar de manera profunda las emociones propias y las de los demás para, así, reconocer de qué sentimiento hacen parte y permitir la reflexión y cuestionamiento de las causas o consecuencias de dicho sentimiento. Vincula las siguientes subcategorías: habilidad para designar diferentes emociones y reconocer las relaciones entre la palabra y el propio significado de la emoción; habilidad para entender las relaciones entre las emociones y las diferentes situaciones a las que obedecen; y la habilidad para reconocer las transiciones de unos estados emocionales a otros.

- Regulación Emocional: Es la habilidad que abarca un proceso más amplio y complicado en cuanto a la IE, ya que no solo se remite a la capacidad de reconocer y comprender, sino que, además, se trata de ser flexible frente a los sentimientos de los demás y los propios para crear situaciones de reflexión consciente que permitan la resolución de problemas. Dentro de sus subcategorías se destacan la habilidad para estar abierto tanto a los estados emocionales positivos como negativos y la habilidad para reflexionar sobre las emociones y determinar la utilidad de la información que estas brindan.

De otra parte, el desarrollo emocional es un concepto que se ha ido tejiendo a través del tiempo y entrelazando con el desarrollo evolutivo. Brinda la posibilidad de visibilizar características específicas en los comportamientos humanos y reafirma la etapa infantil como una de las más importantes para lograr una comprensión de dichos comportamientos. En este sentido, Da Silva y Calvo (2014) plantean que "el desarrollo emocional, así como las demás funciones psíquicas, se determina por la relación entre el sujeto y la cultura" (p. 11).

Cuando se menciona el desarrollo emocional se pueden abarcar aquellos procesos necesarios en la infancia para conseguir un logro que será evidenciado a corto y largo plazo. En los niños es común encontrar respuestas emocionales a partir de reflejos arcaicos, es decir, que de manera natural y a través de estímulos externos crean acciones, las cuales son producto de las emociones, funcionan de manera preventiva y dan lugar a la expresión y a nuevos descubrimientos del infante (Wallon, 2000).

De ahí que se reitere la importancia de cultivar la infancia desde expresiones verbales, corporales y sensitivas, de manera armónica, para un desarrollo integral que proporcione elementos positivos para la vida futura. Shapiro (1997) destaca lo fundamental de las emociones en la infancia, guiadas favorablemente, para un correcto desarrollo en la vida futura. El desarrollo emocional no se da estático y se encuentra en todo tipo de interacción social, es allí donde es necesario referenciar la familia, los estilos parentales y su importancia dentro del entorno cultural como agente primordial en todo proceso 
de aprendizaje.

\section{Metodología}

En el desarrollo de este proyecto investigativo se utilizó el paradigma cualitativo desde un enfoque interpretativo o hermenéutico, teniendo en cuenta la comprensión de las prácticas sociales y el significado que estas aportan a la sociedad (Pievi \& Bravin, 2009). Se considera que el enfoque metodológico más pertinente es el diseño narrativo. Asimismo, se utilizó el estudio de caso como método de análisis y reflexión. Al respecto Martínez (2006) plantea:

El método de estudio de caso es una estrategia metodológica de investigación científica, útil en la generación de resultados que posibilitan el fortalecimiento, crecimiento y desarrollo de las teorías existentes o el surgimiento de nuevos paradigmas científicos; por lo tanto, contribuye al desarrollo de un campo científico determinado (p. 189).

Se delimitó el estudio de caso a una familia que habita la zona urbana del municipio de Gámeza Boyacá, los integrantes de la familia son oriundos de dicho municipio. Es un tipo de familia nuclear conformada por padre, madre, hijo mayor de cinco años e hijo menor de cuatro años. Teniendo en cuenta el propósito de la investigación, la recolección de información se realizó a partir de distintas técnicas como la observación participante y la entrevista semiestructurada. Según Bautista (2011) en la observación participante se mantiene una relación activa desde la comunicación entre el observador y los observados, lo que permite esclarecer de manera concreta la realidad en lo cotidiano de los otros, así mismo se es flexible en dicho proceso.

Se trabajó a partir de instrumentos como el diario de campo, la lectura de cuentos afines a las categorías y subcategorías determinadas y trabajos realizados en casa a partir de guías de aprendizaje diseñadas para el estudiante. El análisis de la información recogida estuvo centrado en dos categorías, cada una con sus respectivas subcategorías. 1) Categoría Inteligencia Emocional: subcategorías comprensión emocional, percepción emocional y regulación emocional. 2) Categoría estilos parentales: subcategorías autoritario, democrático y permisivo.

\section{Resultados}

\section{Inteligencia Emocional}

Percepción y Expresión Emocional. Las interacciones que se viven cotidianamente entre los integrantes de la familia posibilitan tanto formas de expresión emocional como la percepción de estas desde el lenguaje corporal, verbal, gráfico y expresivo. Se hace evidente, también, en las situaciones que se presentan y afectan el estado emocional con expresiones de alegría, tristeza y sorpresa. Estas experiencias favorecen el desarrollo emocional del nińo al establecer vinculaciones con la cotidianidad para reconfigurar formas de relación con la familia y con las personas que le rodean. 
En este sentido, la familia como primer agente socializador del niño requiere tener en cuenta la relación entre la expresión y la actitud en las diferentes manifestaciones de las emociones con los integrantes de la familia. En las interacciones cotidianas se manifiestan acciones emocionales, verbales y corporales específicas que, a su vez, se configuran en modelos que los niños van apropiando para expresar lo que sienten y reconocer tales patrones en las demás personas. Lo anterior, incide en los años de desarrollo posteriores en las interacciones con otras personas del entorno. En situaciones de conflicto se asumen expresiones como gritos, palabras no adecuadas, movimientos acelerados y tensión en el ambiente. Teniendo en cuenta la importancia de la comunicación y la verbalización emocional, lo anterior da cuenta de que:

Los adultos que verbalizan sus emociones asiduamente incrementan la atención del niño hacia éstas. Según diversos estudios recogidos por Palacios, Marchesi y Coll (1990), cuando los progenitores verbalizan con frecuencia sus propias respuestas empáticas, sus hijos presentan una tendencia a mostrar mayor atención a esos procesos y, en consecuencia, incrementan la comprensión de cómo actúan (Gonzales \& Gomariz, 2020, p. 284).

De igual forma, los padres de familia reconocen en sus hijos las emociones primarias, como la tristeza y la alegría, frente a situaciones cotidianas. También notan cambios repentinos, por ejemplo, cuando los niños pasan de la tranquilidad al enojo, en donde la reacción tanto física como verbal puede tornarse agresiva.

El niño participante asocia las emociones a una acción definida. Por ejemplo, la felicidad en respuesta a situaciones en donde recibe detalles. Asimismo, reconoce algunas expresiones y acciones emocionales en su familia y las representa a través de dibujos y diálogos con su maestra como se evidencia en las figuras 1 y 2.

\section{Figura 1}

¿Estoy Triste o Estoy Contento?

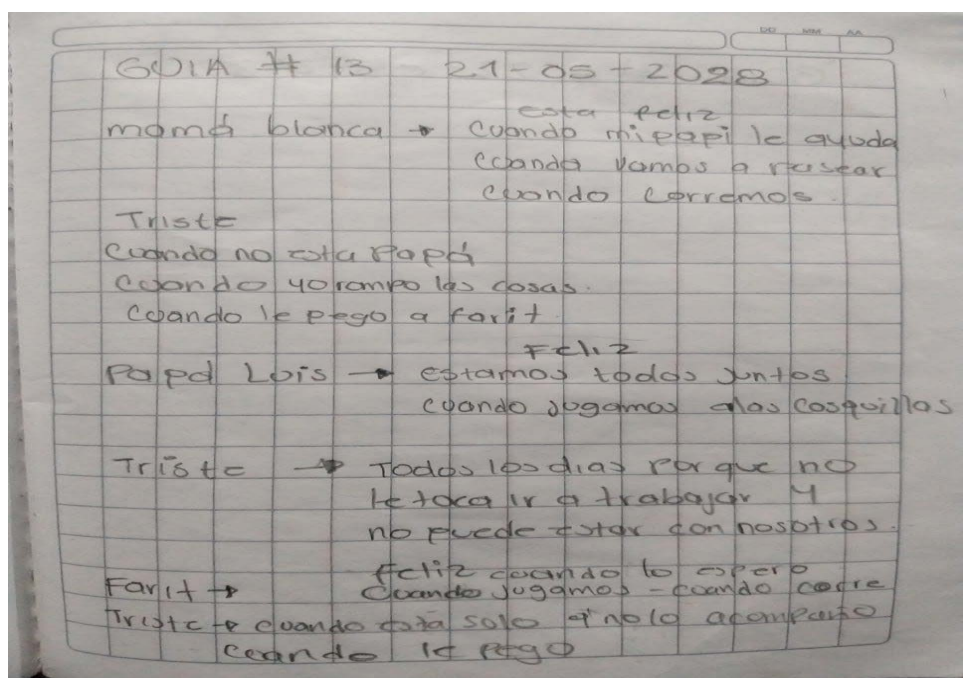


Nota: material extraído del cuaderno de tareas del participante.

Shapiro (1977) indica que "aprender a identificar y transmitir emociones es una parte importante de la comunicación ... Pero apreciar las emociones de los demás constituye una capacidad del CE (coeficiente emocional) igualmente importante, particularmente para el desarrollo de relaciones íntimas y satisfactorias” (p.1 55). En este caso, a partir de la narración del cuento ¿estoy triste o estoy contento? de la autora Antonella Abatiello, el niño realizó una asociación de dos estados de ánimo con cada uno de los miembros de su familia. Así, el participante, según situaciones que percibe en casa, afirma en qué momentos cada integrante de la familia muestra que está triste o contento; la madre de familia transcribe la información dada por el niño sin generar modificaciones. De esta manera se evidencia que el niño logra percibir, reconocer y expresar emociones de las demás personas.

Figura 2

Emoticones en Mi Familia

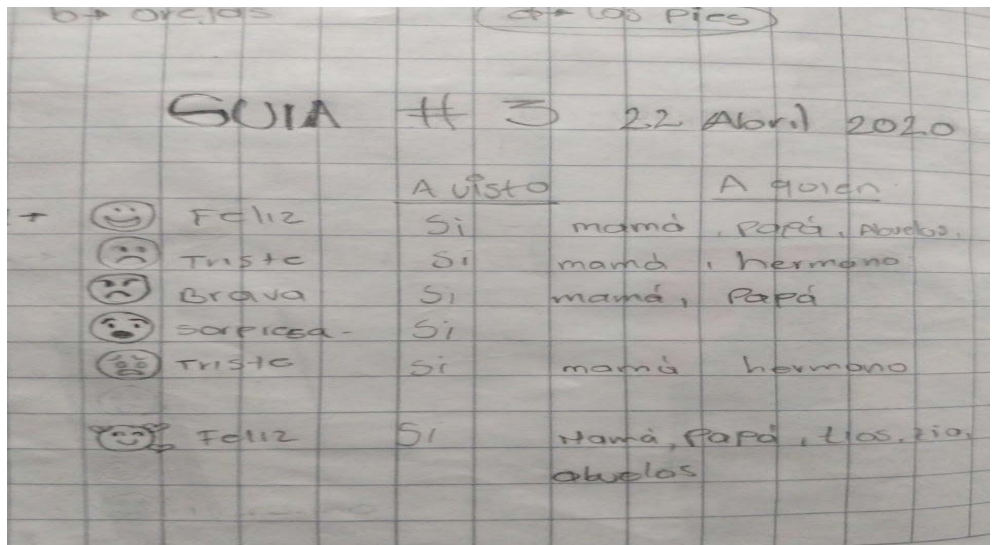

Nota: material extraído del cuaderno de tareas del participante.

En las anteriores figuras se evidencia que el niño reconoce la expresión de emociones como la tristeza y la alegría, y los motivos que las provocan en cada uno de los integrantes de la familia, lo que favorece el reconocimiento de actitudes físicas de las reacciones emocionales. Según Aresté (s.f.) "Desde los dos años, los niños son capaces de reconocer algunas emociones en los demás y dar una respuesta a estas. Y, a los cuatro años los infantes son capaces de adaptar las emociones a cada situación" (p. 22). La expresión de las emociones en los integrantes de la familia permite que el nińo establezca una correspondencia entre la actitud, la emoción y los motivos que provocan estas emociones, lo que favorece la comunicación con las personas que le rodean. Además, su reconocimiento posibilita tener el control de sus emociones.

Comprensión emocional. Desde esta categoría se hace evidente que en el grupo familiar las emociones no son muy tomadas en cuenta, de manera consciente, dentro 
de las interacciones cotidianas. Se desconoce un significado específico sobre lo emocional, pero se realizan asociaciones con los estados de ánimo a partir de una situación en particular. Reconocen que tales estados se transmiten y tienen influencia sobre sus hijos. Autores como López (2011) o Bizquerra (2010) concuerdan en que las emociones responden a realidades de la cotidianidad expresadas de diferentes maneras, ya sea verbal y/o corporal, además, evidencia una estrecha correlación con uno o más sujetos. Tal planteamiento pone de relieve que al experimentar las emociones en el diario vivir se puede asumir que no debería ser desconocido hablar de ellas.

Algunos autores señalan que las neuronas espejo hacen posible la comprensión de los estados emocionales de otras personas. (...) Esto lleva a ponerse en su lugar y experimentar un estado emocional similar. Las deficiencias en el funcionamiento de las neuronas espejo podrían suponer una incapacidad para ponerse en el lugar del otro. Y, por tanto, la incapacidad para experimentar empatía y compasión. (Aguado, 2005, citado en Bizquerra, 2010, p. 111)

En este sentido, los padres de familia identifican, en ellos mismos, algunas emociones de manera superficial y perciben cambios en la interacción con sus hijos. De esta manera, les es posible acercarse a la comprensión de emociones propias, lo que lleva al reconocimiento de cambios emocionales. Sin embargo, no existe una reflexión sobre la incidencia de estos cambios en el desarrollo emocional del niño. Del mismo modo, los padres del participante manifestaron que las emociones negativas y positivas influyen en las interacciones con los integrantes de la familia. En cuanto a las emociones negativas están relacionadas con situaciones laborales, exceso de trabajo en el hogar y responsabilidades académicas de los hijos, lo cual induce a menores niveles de tolerancia y paciencia, presencia de indisposición y estados de ánimo alterados o de enojo. Todo lo anterior influye en los aprendizajes cotidianos, en especial de los nińos.

Al respecto, Goleman (citado en Herrera, 2015) plantea que "es más sencillo enseñar a niños todas las habilidades de la inteligencia emocional durante el periodo en que está conformándose su sistema de circuitos neuronales, que tratar de modificarlos luego" (p. 33). En consecuencia, resulta oportuno que la madre participante realice reflexiones acerca de la manera como se relaciona con su hijo, pues esto podría ser una oportunidad para revisar patrones de crianza y garantizar a tiempo una infancia segura.

Es relevante para el núcleo familiar el reconocimiento y la representación de emociones primarias como la alegría, tristeza y enojo. La figura 3 muestra la representación de las emociones del niño y sus ubicaciones en un cuadro comparativo que hace énfasis en cuáles emociones le gusta sentir y cuáles no, estableciendo relación entre la acción y la emoción que la representa. 
De otra parte, autoras como González y López (2011) plantean la importancia de que los adultos que guían procesos de aprendizaje, reconozcan las potencialidades de utilizar aquellas circunstancias generadoras de emociones negativas en los niños para preguntar y analizar acerca de estas, pues esto permite que el niño distinga y comprenda mejor sus propias emociones.

A partir de las distintas interpretaciones se puede concluir que los adultos relacionados a esta investigación muestran un nivel bajo en su comprensión emocional, lo cual incide de manera específica en sus hijos quienes presentan dificultad en esta habilidad.

\section{Figura 3}

Emoción Que Me Gusta Tener y Emoción Que No Me Gusta Tener

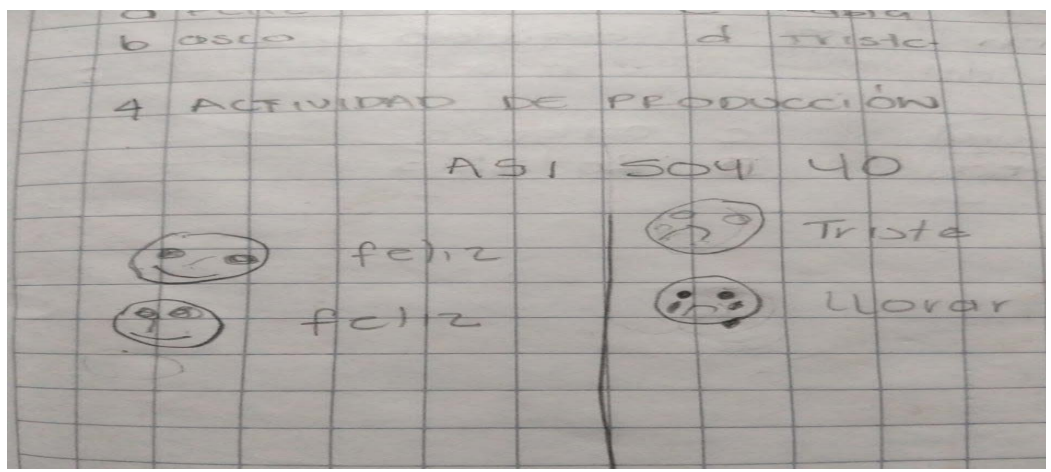

Nota: material extraído del cuaderno de tareas del participante.

Regulación emocional. Para retomar esta categoría, el tópico de conversación es escenarios de conflicto familiar y la manera en que usualmente estos se resuelven. Se evidenció que los adultos interpretan desde una mirada personal y especifica su sentir dentro del núcleo familiar. Situaciones como el desorden, el estado de ánimo, el cansancio físico y mental, no lograr objetivos establecidos y el desequilibrio en las responsabilidades del hogar generan reacciones que tienen como consecuencia la dificultad en la regulación emocional tanto de los adultos como de los niños.

En este sentido, "la verbalización de las emociones facilita la regulación emocional del niño. Le permite tomar conciencia de ellas y aumentar su habilidad para controlar las situaciones, para buscar soluciones a los problemas y para reparar sus estados de ánimo alterados" (Fernández-Berrocal \& Ramos, 2005, citado en Gonzales \& Gomariz, 2020, p. 284).

Conviene subrayar la importancia de la comunicación en las situaciones de conflicto dentro y fuera del hogar. Aunque el diálogo es el mediador para resolver todo tipo de conflictos, es reiterativo encontrar situaciones en donde la comunicación no funciona de la misma manera hacia los niños, más bien esta se da en sentido unidireccional y la gran mayoría de veces es ejercida por los adultos. Al respecto, es 
importante tener en cuenta el papel de la expresión y comunicación en la regulación emocional, pues "las emociones son respuestas internas que desencadenan acciones y conductas. La forma como chicos aprenden a expresarlas los puede llevar al éxito o al fracaso en la comunicación" (Gonzales \& López, 2011, p. 44).

Por otra parte, situaciones de vigilancia y control que el adulto ejerce sobre el niño ocasionan estados de miedo y angustia evitando expresiones emocionales que ejercen autocontrol. Al respecto, Gonzales y López (2011) refieren que "cuando se habla de manejar las emociones no se hace referencia a la necesidad de reprimirlas, sino a la capacidad de dirigirlas y equilibrarlas, expresándolas en su justa proporción” (p.43). Aunque dichas acciones son transmitidas por los integrantes de la familia, se hacen evidentes en el niño otras formas de interacción con pares externos al hogar, como se logra evidenciar en la figura 4.

Figura 4

Mis Amigos

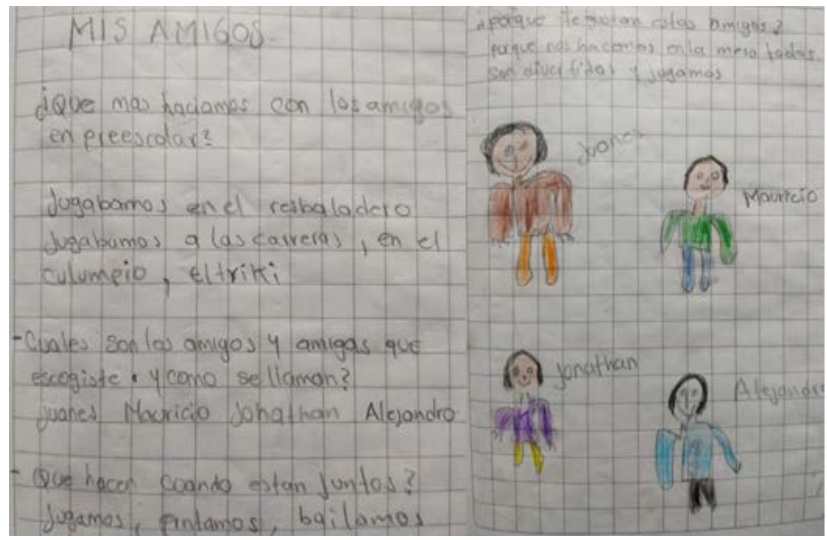

Nota: material extraído del cuaderno de tareas del participante.

Teniendo en cuenta la figura anterior y en específico el dibujo relacionado a los amigos del niño, se puede observar orden en la distribución de los personajes en cuanto a la hoja y, aunque son posibles distintas variaciones para lograr dar una definición especifica en un dibujo, al analizar la imagen se evidencia que una de las emociones allí plasmadas es la alegría que desde la perspectiva de Ferreros (citado en Aguayo et al., 2016) se plasma como "un conjunto armonioso, con buena organización de los elementos, colores variados y cálidos" (p. 31). También, en los relatos del nińo se encuentra similitud sobre las emociones positivas asociadas a la importancia de sus amigos y su relación con sentir con interacciones significativas. Asimismo, al abordar la regulación emocional se hace necesario tener en cuenta todo tipo de relaciones sociales. Dichas interacciones dan inicio en el entorno familiar, como indica Gonzales y López (2011) al rescatar un sin fin de habilidades propias de un buen desarrollo de IE, lo que da paso a una buena socialización y apropiación del mundo. En concordancia, Shapiro (1997) realiza un análisis sobre la importancia de 
la amistad como relación social y emocional teniendo en cuenta la edad en el niño, como se menciona a continuación:

En la época en que un niño tiene siete u ocho años, comienza a alejarse de la influencia de sus padres y, cada año que pasa, mira con más frecuencia hacia sus compañeros de clase y amigos como fuente de afecto, aprobación y apoyo. Aunque en la familia el apoyo emocional es algo que se da por sentado, entre grupos de nińos se trata de una recompensa que se gana. El camino hacia dicha recompensa se vislumbra en su mayor parte a través de la capacidad emocional y social del niño. Según Sullivan, la amistad entre niños imprime hábitos de por vida en la relación con los demás, así como un sentido de autoestima casi igual al que se desarrolla a través del amor y el cuidado de los padres. De manera inversa, cuando un niño carece de amigos o de la aceptación de sus pares, en particular durante los ańos de la escuela primaria, carga con cierto sentido de lo incompleto y de insatisfacción, a menudo a pesar de logros significativos (p. 111).

Es necesario aclarar que la regulación emocional es una habilidad que se debe trabajar desde temprana edad. Sin embargo, es más factible que sea el adulto quien regule sus emociones para aportar significativamente en los procesos de modelamiento del niño.

\section{Estilos Parentales}

Los resultados muestran que en las interacciones familiares cotidianas prevalecen los estilos parentales autoritario y permisivo. Por un lado, el estilo autoritario se hace visible desde el control de padres a hijos y las correcciones continuas frente a su forma de hablar e interactuar con protocolos o formalismos en el saludo, la despedida o las respuestas afirmativas y negativas. El desconocimiento de las habilidades propias de la edad del niño para realizar actividades del hogar no genera oportunidades en las que él pueda tomar sus decisiones. Estas actitudes impiden su fluidez verbal y bloquean distintas formas para comunicar sus experiencias cotidianas. El adulto manifiesta su control a través de expresiones faciales y verbales. Al respecto, es importante mencionar que cada estilo parental tiene una repercusión diferente en el desarrollo de sus hijos. "a mayor estilo autoritario o permisivo, menor rendimiento en componentes de empatía y desempeño emocional general” (Henao \& García, 2009, p. 798).

En este sentido, cuando el niño está inmerso en este tipo de ambientes tiende a repetir patrones de control y autoridad frente a sus pares o en relaciones donde él pueda ejercer algún tipo de poder. Lo anterior, permite relacionar algunas características de padres autoritarios. Al respecto, Henao y García (2009), afirman que:

Este es un patrón muy restrictivo de crianza en el que las personas adultas imponen muchas reglas, esperan una obediencia estricta, rara vez o nunca 
explican al niño o niña por qué es necesario obedecer todas estas regulaciones (...) Los padres y madres autoritarios no son sensibles a los puntos de vista en conflicto de un niño o una niña, esperando en lugar de ello que el niño o niña acepte su palabra como ley y respete su autoridad (p. 788).

Los padres priorizan dentro de las pautas de crianza el castigo en respuesta a las formas de mal comportamiento y desobediencia de los nińos. Este se da de manera verbal y física; omite la justificación de las acciones que no son adecuadas para los adultos.

Por otro lado, el estilo parental permisivo se manifiesta en situaciones donde se conceden al niño objetos materiales a demanda con el fin de suplir el tiempo y las carencias afectivas generadas por los padres, lo cual desconoce otras formas de brindar estímulos que motiven el autorreconocimiento, la confianza en el niño y el fortalecimiento de la comunicación entre padres e hijos. En relación, Jorge y Gonzales (2017) reiteran que en la permisividad los adultos buscan la felicidad y alegría del niño, por lo tanto, suplen toda demanda sin importar cuales sean sus exigencias, aunque también advierten del deseo afectivo hacia los hijos.

Se concluye que la estructura de crianza en la familia estudiada es relevante desde el estilo parental autoritario y los rasgos de permisividad son muy pocos. Jorge y Gonzales (2017) insisten en que las familias no se constituyen totalmente en un solo estilo parental, ya que este puede ser variable y flexible según factores externos. Lo expuesto anteriormente lleva a reflexionar acerca de la importancia que tiene la familia y los estilos parentales que se tejen en cada núcleo familiar, y su incidencia en la percepción, expresión y regulación de las emociones de los hijos, desde las actitudes y expresiones verbales, faciales y corporales; estas influyen en los niños, sus respuestas de sumisión y/o hostilidad y sus interacciones con los demás

\section{Conclusiones}

Las interacciones familiares están determinadas a partir de una voz de mando direccionada por los adultos, quienes son los que guían y toman las decisiones en el hogar. Los niños reafirman las peticiones de sus padres y están sujetos a la variabilidad de estos en cuanto a las dinámicas de la cotidianidad. La autoridad predomina en cada una de sus acciones, para las cuales se resalta el cumplimiento de las labores en el campo, la mina y el hogar. Este ambiente dificulta un adecuado desarrollo emocional para el niño, que influye en la concepción del rol del hombre y la mujer en una familia y también provoca una réplica de comportamientos ofrecidos por parte de los adultos en las interacciones con los demás.

Se reconocen distintas situaciones emocionales para cada sujeto. La madre prioriza la afectación de las acciones cotidianas en las emociones de sus hijos, mientras que el padre revisa la importancia de las emociones propias y no distingue las de sus hijos. Por ello, fue más sencillo que la madre de familia relatara con fluidez sus interacciones 
acompañadas de una carga emocional, mientras que al padre le costó expresarse en relación con las dinámicas en su familia.

Los estilos parentales que se manifiestan en las relaciones familiares, en la mayoría de las situaciones, son el estilo autoritario y, en poca medida, el estilo permisivo, los cuales no favorecen las relaciones de seguridad y confianza en sí mismos de los hijos, que más adelante se verá reflejado en la dificultad para establecer relaciones participativas, comprensivas y armónicas entre padres e hijos. El desarrollo emocional se establece por la relación entre el niño y la cultura, desde experiencias entre los padres e hijos. Estas son la base para formar la personalidad, la cual se construye en la medida en que tales relaciones lleguen a ser participativas y democráticas.

Es importante reconocer las características propias de la edad del niño participante, pues en esta etapa se hace susceptible a los encuentros emocionales, necesita mucho afecto, que sus acciones sean reconocidas y que las acciones que no entiende sean explicadas de manera directa o con ejemplos concretos de su contexto; esto le permite mayor comprensión ante la ambigüedad del querer hacer y el sentir. Es necesario destacar la necesidad de fortalecer la comunicación entre los integrantes de la familia (entiéndase como todos aquellos miembros directos que contienen y aportan en la socialización de los nińos y nińas) como estrategia de convivencia desde la primera infancia. Esta comunicación debe dar confianza, seguridad, y lugar a interacciones que estén mediadas por la apertura para escuchar y ser escuchados.

Resulta fundamental indagar sobre el desarrollo emocional de los padres, su historia de vida, la forma en que fueron criados y las afectaciones, o no, que ellos mismos encuentran en su adultez, para poder revisar la incidencia emocional en sus vidas a partir de las experiencias con sus padres y al mismo tiempo replantear la de ellos con sus hijos. Esta sería una manera más concreta de reconocer falencias culturales que se encuentran dentro de la crianza y, que, de manera inconsciente, se siguen transmitiendo, replicando una y otra vez situaciones de dolor, tristeza, resentimiento y, sobre todo, la imposibilidad de hablar de ellas, soltar y sanar.

También, se hace necesario continuar investigaciones afines al tema emocional y avanzar en programas institucionales a nivel local, municipal y nacional que visibilicen las emociones como prioridad. Así como desarrollar propuestas que permitan educar emocionalmente a las familias, de manera constante y clara, para que los mismos currículos educativos utilicen la educación emocional no como una estrategia de transversalización, sino como la base de toda educación social y cognitiva.

Ahora bien, es preciso encontrar discusiones y reflexiones en autoras como Federici (2004), Medina (2012) o Esteban (2017) que, en su gran mayoría, develan la importancia del papel que tiene la mujer en el cuidado hacia la sociedad, ya que desde culturas antiguas han sido custodias de saberes y guías espirituales gracias a su sabiduría y entendimiento desde su sensibilidad y entrega. Es allí donde se centra 
un gran cuestionamiento, si cultural e históricamente se le ha atribuido a la madre el cuidado del hogar y de los hijos, ¿por qué no hemos trabajado como conjunto social para lograr el cuidado pertinente y de calidad hacia las madres? No se trata solo de crear políticas públicas y ahondar en leyes que difícilmente se cumplen, se hace necesario que la humanidad asuma como propósito un espacio que contenga a las madres y las alimente emocionalmente. Esto se traduciría en mujeres menos fracturadas, incluso mentalmente, y crearía procesos armónicos en aquellos infantes que un día serán adultos y aportaran significativamente a una transformación social equilibrada y empática.

\section{Referentes}

Aguayo, P., Herrera, B., Ortiz, M., \& Vallejos, C. (2016). El dibujo infantil: una herramienta para potenciar las diferentes áreas de desarrollo psicoemocional y creativo en niños y niñas de 4 a 6 años [Trabajo de grado. Universidad de Concepción Campus Los Ángeles]. http://repositorio.udec.cl/jspui/handle/11594/2287

Aresté, J. (s.f.). Las emociones en educación infantil: sentir, reconocer y expresar [Trabajo de grado, Universidad Internacional la Rioja] https://n9.cl/0x9wd

Bautista, N. (2011). Proceso de la investigación cualitativa. Epistemología, metodología y aplicaciones. El Manual Moderno.

Bisquerra, R. (2010). Psicopedagogía de las emociones. Editorial Síntesis.

Capano, A., \& Ubach, A. (2013). Estilos parentales, Parentalidad Positiva y Formación de Padres. Ciencias Psicológicas, 7(1), 83 -95. http://www.scielo.edu. uy/pdf/cp/v7n1/v7n1a08.pdf

Da Silva, R., \& Calvo, S. (2014). La actividad infantil y el desarrollo emocional en la infancia. Revista Intercontinental de Psicología y Educación, 16(2), 9-30.

Esteban, M. (2017). Los cuidados, un concepto central en la teoría feminista: aportaciones, riesgos y diálogos con la antropología. Quaderns-e de l'Institut Català d'Antropologia, 22(2), 33-48. https://raco.cat/index.php/QuadernseICA/ article/view/333111

Federici, S. (2004). Calibán y la bruja. Mujeres, cuerpo y acumulación originaria. Traficantes de Sueños. https://www.traficantes.net/sites/default/files/pdfs/ Caliban\%20y\%20la\%20bruja-TdS.pdf

Fernández, P., \& Extremera, N. (2005). La Inteligencia Emocional y la educación de las emociones desde el Modelo de Mayer y Salovey. Revista Interuniversitaria de Formación del Profesorado, 19(3), 63-93.

Fernández-Berrocal, P., Extremera, N., Palomera, P., Ruiz-Aranda, D., Salguero, J., \& Cabello, R. (Coords.). (2015). De la neurona a la felicidad. Diez propuestas 
desde la inteligencia emocional. Fundación Botín.

García, E. (2017). Formación del profesorado en Educación Emocional [Tesis doctoral, Universidad de Barcelona]. https:/www.tesisenred.net/bitstream/ handle/10803/454728/EGN_TESIS.pdf?seque

Gonzales, M., \& Gomariz, M. (2020). La verbalización de las emociones en educación infantil. Estudios sobre educación. 38, 279-302. https://doi. org/10.15581/004.38.279-302

Gonzales, M., \& López, M. (2011). Haga de su hijo un gigante emocional. Herramientas para enseñar a controlar y orientar sus emociones. Ediciones Gamma.

Gutiérrez, F. (2019). El concepto de familia en Colombia: una reflexión basada en los aportes de la antropóloga Virginia Gutiérrez sobre la familia colombiana en el marco de la doctrina constitucional. Revista Temas Socio-Jurídicos, 38(76), 130154. https://doi.org/10.29375/01208578.3589

Henao, G., \& García, M. (2009). Interacción familiar y desarrollo emocional en niños y niñas. Revista Latinoamericana de Ciencias Sociales, Niñez y Juventud, 7(2), 785-802.

Herrera, A. (2015). La educación emocional desde la etapa preescolar. Revista para el Aula IDEA, (16), 33-35.

Jorge, E., \& González, C. (2017). Estilos de crianza parental: una revisión teórica. Informes Psicológicos, 17(2), 39-66. http://dx.doi.org/10.18566/infpsic.v17n2a02

López, B. (2011, 5 de abril). Cómo educar las emociones de nuestros hijos [Conferencia]. Programa "Brújula en familia", España.

Martínez, A. (2010). Pautas de crianza y desarrollo socioafectivo en la infancia. Revista diversitas-perspectivas en psicología, 6(1). 165-193. http://www.scielo.org. co/pdf/dpp/v6n1/v6n1a09.pdf

Martínez, P. (2006). El método de estudio de caso: estrategia metodológica de la investigación científica. Pensamiento \& Gestión, (20), 165-193.

Medina, R. (2012). Sentir la historia. Propuestas para una agenda de investigación feminista en la historia de las emociones. Arenal, 19(1). 161-199. https://doi. org/10.30827/arenal.v19i1.1412

Pievi, N., \& Bravin, C. (2009). Documento metodológico orientador para la investigación educativa. Ministerio de Educación de la Nación. http://repositorio. educacion.gov.ar:8080/dspace/handle/123456789/96341

Shapiro, L. (1997). Inteligencia emocional de los niños. Vergara Editor.

Wallon, H. (2000). La evolución psicológica del niño, (1 $1^{\mathrm{a}}$ ed.). Biblioteca de Bolsillo 